\title{
Statistical analyses and quality of individual participant data network meta-analyses were suboptimal: a cross-sectional study
}

Ya Gao ${ }^{1 \dagger}$, Shuzhen Shi ${ }^{1+}$, Muyang $\mathrm{Li}^{2}$, Xinyue Luo ${ }^{2}$, Ming Liu' ${ }^{1}$ Kelu Yang ${ }^{3}$, Junhua Zhang ${ }^{4}$, Fujian Song ${ }^{5 *}$ and Jinhui $\operatorname{Tian}^{1,6^{*}}$

\begin{abstract}
Background: Network meta-analyses using individual participant data (IPD-NMAs) have been increasingly used to compare the effects of multiple interventions. Although there have been many studies on statistical methods for IPD-NMAs, it is unclear whether there are statistical defects in published IPD-NMAs and whether the reporting of statistical analyses has improved. This study aimed to investigate statistical methods used and assess the reporting and methodological quality of IPD-NMAs.
\end{abstract}

Methods: We searched four bibliographic databases to identify published IPD-NMAs. The methodological quality was assessed using AMSTAR-2 and reporting quality assessed based on PRISMA-IPD and PRISMA-NMA. We performed stratified analyses and correlation analyses to explore the factors that might affect quality.

Results: We identified 21 IPD-NMAs. Only 23.8\% of the included IPD-NMAs reported statistical techniques used for missing participant data, $42.9 \%$ assessed the consistency, and none assessed the transitivity. None of the included IPD-NMAs reported sources of funding for trials included, only 9.5\% stated pre-registration of protocols, and 28.6\% assessed the risk of bias in individual studies. For reporting quality, compliance rates were lower than $50.0 \%$ for more than half of the items. Less than 15.0\% of the IPD-NMAs reported data integrity, presented the network geometry, or clarified risk of bias across studies. IPD-NMAs with statistical or epidemiological authors often better assessed the inconsistency $(P=0.017)$. IPD-NMAs with a priori protocol were associated with higher reporting quality in terms of search $(P=0.046)$, data collection process $(P=0.031)$, and syntheses of results $(P=0.006)$.

Conclusions: The reporting of statistical methods and compliance rates of methodological and reporting items of IPD-NMAs were suboptimal. Authors of future IPD-NMAs should address the identified flaws and strictly adhere to methodological and reporting guidelines.

Keywords: Network meta-analysis, Individual participant data, Statistical analysis, Methodological quality, Reporting quality

\footnotetext{
* Correspondence: tianjh@|zu.edu.cn; fujian.song@uea.ac.uk

${ }^{\dagger}$ Ya Gao and Shuzhen Shi contributed equally to this work.

${ }^{5}$ Public Health and Health Services Research, Norwich Medical School,

University of East Anglia, Norwich NR4 7TJ, UK

'Evidence-Based Medicine Center, School of Basic Medical Sciences, Lanzhou

University, No.199, Donggang West Road, Lanzhou City 730000, Gansu

Province, China

Full list of author information is available at the end of the article
}

(c) The Author(s). 2020 Open Access This article is licensed under a Creative Commons Attribution 4.0 International License, which permits use, sharing, adaptation, distribution and reproduction in any medium or format, as long as you give appropriate credit to the original author(s) and the source, provide a link to the Creative Commons licence, and indicate if changes were made. The images or other third party material in this article are included in the article's Creative Commons licence, unless indicated otherwise in a credit line to the material. If material is not included in the article's Creative Commons licence and your intended use is not permitted by statutory regulation or exceeds the permitted use, you will need to obtain permission directly from the copyright holder. To view a copy of this licence, visit http://creativecommons.org/licenses/by/4.0/. The Creative Commons Public Domain Dedication waiver (http://creativecommons.org/publicdomain/zero/1.0/) applies to the data made available in this article, unless otherwise stated in a credit line to the data. 


\section{Background}

Meta-analysis based on individual participant data (IPD) from randomized controlled trials (RCTs) [1, 2] is considered the "gold standard" of meta-analyses. The use of IPD in meta-analysis offers more flexibility in the investigation of patient-level moderators [3], allows the standardization of outcome definitions and analyses across studies $[4,5]$, and helps improve the quantity and quality of data [6-8]. Network meta-analysis (NMA), also known as mixed treatment comparison or multiple treatments comparison meta-analysis, is a statistical method to directly and indirectly compare the effects of two or more treatments and allows ranking of different treatments [9-12]. Although NMAs usually include aggregate data from RCTs [13], there are many advantages to incorporating IPD into NMAs, including more appropriate investigation of heterogeneity or inconsistency by using advanced modeling strategies to explore subjectlevel covariates [14-16], and identifying interactions of patient-level effect modifiers [17-19].

Previous studies have evaluated the statistical methods, methodological and reporting characteristics of IPD meta-analyses [2, 20]. A comprehensive scoping review found that indirect comparisons using IPD often failed to report key statistical methods [3], which may lead to biased conclusions. Several studies also assessed the methodological quality and the conduct of published NMAs [21-25]. But no studies have focused solely on the reporting and methodological quality of IPD-NMAs. Researchers have recognized that the use of IPD in NMAs may yield the most trustworthy evidence to inform clinical decision-making [26]. However, when IPDNMAs have methodological flaws, they could threaten the validity of the results and thus mislead clinical decision-making [16, 27]. Although there have been many studies on statistical methods for IPD-NMAs [26, $28,29]$, it is unclear whether there are statistical defects in published IPD-NMAs and whether the reporting of statistical analyses has improved.

The objectives of this study were to explore the general characteristics, statistical analysis methods used, reporting quality, and methodological quality of IPDNMAs and to identify study-level variables that were associated with the methodological and reporting quality.

\section{Methods}

\section{Literature search}

A comprehensive literature search was conducted on PubMed, Embase.com, Cochrane Library, and Web of Science using the following items: "network metaanalysis", "mixed treatment comparison meta-analysis", "mixed treatment meta-analysis", "multiple treatment comparison meta-analysis", "multiple treatment metaanalysis", "indirect comparison", "individual patient", "individual participant", and "patient level". The detailed search strategy of PubMed is presented in Additional file 1: Appendix Word 1. We performed the initial search on December 13, 2018, and updated the search on June 3, 2019. We also manually retrieved references of included IPD-NMAs and relevant reviews.

\section{Eligibility criteria}

Because NMAs using IPD from RCTs provide the most valid results, we included IPD-NMAs of RCTs that evaluated the clinical effects of three or more interventions for patients in any clinical conditions. There were no restrictions on publication year and language.

Studies including the following were excluded: (1) NMAs did not incorporate IPD; (2) pairwise metaanalyses of IPD; (3) IPD-NMAs did not focus on health care interventions such as etiology and diagnosis; (4) studies that only applied simulated treatment comparison [30], adjusted indirect comparisons [31], or matching adjusted indirect comparisons (MAICs) [32] failing to preserve within-study randomization; and (5) methodological studies, review protocols, abstracts, conference proceedings, and letters to editors.

\section{Study selection}

We used EndNote X8 (Thomson Reuters (Scientific) LLC Philadelphia, PA, USA) to manage the retrieved records. Two review authors (Y.G. and S.Z.S.) independently reviewed titles and abstracts identified through the electronic search. Full reports of any potentially relevant papers were retrieved for further assessment of eligibility. If an IPD-NMA has been updated, we would only include the latest version. Differences of opinion were settled by consensus or referral to a third review author (F.J.S. or J.H.T.).

\section{Data extraction}

We developed a data extraction form using Microsoft Excel 2016 (Microsoft Corp, Redmond, WA, www. microsoft.com) to abstract data on general characteristics and statistical analysis methods used, including publication year, first author, country of corresponding authors, number of authors, journal name, whether a statistician or epidemiologist (based on the author's current academic position) was involved, whether IPDNMAs had a priori protocol, funding source (industry, non-industry, unfunded, or not reported), the topic of interest, number of trials included, number of participants included, number of interventions included, format of data included in analysis (individual participant data, aggregate data), whether used Bayesian method or Frequentist method, 1-stage or 2-stage process, statistical techniques used for missing data, methods used to assess heterogeneity, and methods used to assess 
consistency and transitivity. We piloted the data extraction on a random sample of five included studies and achieved consistency in data item interpretations. Then, four trained authors (Y.G., S.Z.S., M.Y.L., and X.Y.L.) abstracted data from the included IPD-NMAs, and another two reviewers (J.H.Z. and J.H.T.) checked the data. Disagreements were resolved by discussion.

\section{Reporting and methodological quality assessment}

The reporting quality of the included IPD-NMAs was evaluated according to the Preferred Reporting Items for Systematic Review and Meta-Analyses of individual participant data (PRISMA-IPD) statement, which is a checklist of 31 items aimed to improve the completeness and transparency of reporting of systematic reviews (SRs) and meta-analyses of individual participant data [1]. To identify the important information that should be reported in a network meta-analysis, we also applied five supplemental items of Preferred Reporting Items for Systematic Reviews and Meta-analyses extension (PRISMA-NMA) statement (S1. Geometry of the network, S2. Assessment of inconsistency, S3. Presentation of network structure, S4. Summary of network geometry, S5. Exploration for inconsistency) [33]. Each item was rated with "yes" (total compliance), "partial" (partial compliance), or "no" (noncompliance) [24, 34].

We used the Assessment of Multiple Systematic Reviews-2 (AMSTAR-2) tool to assess the methodological quality of the included IPD-NMAs [35]. This tool assesses the methodological quality of SRs across 16 domains, among which seven are critical [35]. Each domain can be rated as "yes" (item fully addressed), "no" (item not addressed), or "partial yes" (item not fully addressed). According to the critical domains, the overall confidence of the quality of each review can be classified as high, moderate, low, or critically low [35]. The quality assessment was conducted by one reviewer (Y.G., S.Z.S., M.Y.L., and X.Y.L.) and verified by another. Possible disagreements were resolved by consensus or with the consultation of a third party (F.J.S. or J.H.T.).

\section{Data analysis}

We applied frequency and percentage to present categorical variables and median and interquartile range (IQR) to present continuous variables. For individual items of reporting and methodological quality, the compliance rate was computed with the number of items acquired "yes" and the total number of the included IPD-NMAs. Then, we created Radar maps and bubble plots using Microsoft Excel 2016 (Microsoft Corp, Redmond, WA, www.microsoft.com) to present the compliance rates. We classified the included IPD-NMAs according to the following characteristics: with or without a statistician or epidemiologist, with or without a priori protocol, non-industry or industry funding, using a Bayesian or a Frequentist method, and using the 1stage process or the 2-stage process. We then performed Fisher's exact test to compare the compliance of each PRISMA-IPD, PRISMA-NMA, and AMSTAR-2 item by the above characteristics. The analyses were conducted in Stata (13.0; Stata Corporation, College Station, Texas, USA), and the statistical level of significance was set at $P<0.05$.

To investigate whether the reporting quality was associated with the methodological quality of IPD-NMAs, we also performed the correlation analysis. We computed the number of items acquired "yes" of PRISMA-IPD and AMSTAR-2 for each IPD-NMA. Then, we conducted the Shapiro-Wilk test and created the Q-Q normal map to evaluate the normality of numbers of items acquired "yes" of PRISMA-IPD and AMSTAR-2 [22, 24], and results indicated that they were normally distributed (Additional file 1: Appendix Word 2). Therefore, we performed the Pearson correlation analysis using Stata (13.0; Stata Corporation, College Station, Texas, USA) to explore the relationship between fully reported PRISMA-IPD items and AMSTAR-2 items.

\section{Results}

\section{Literature search}

The search of electronic databases yielded 1376 records, and we identified five additional records through manually checking the references of relevant reviews. After removing duplicates and screening titles and abstracts, we identified 107 reports for the full-text assessment. We further excluded 9 aggregate NMAs, 39 MAICs, 6 indirect comparisons, 31 methodological studies, and 1 IPD meta-analysis. Eventually, there were 21 IPD-NMAs that met our eligibility criteria (Fig. 1). A list of the included IPD-NMAs is shown in Additional file 1: Appendix Word 3.

\section{General characteristics of included IPD-NMAs}

The first IPD-NMA was published in 2007, and the remaining were all published since 2010. The USA (9, 42.9\%) published the largest number of IPD-NMAs, followed by the UK $(7,33.3 \%)$ and France (3, 14.3\%). A wide range of different diseases was studied in the included IPD-NMAs (Fig. 2). All the 21 IPD-NMAs were conducted by four or more authors, including eight IPDNMAs that involved 11 or more authors. Seven (33.3\%) IPD-NMAs had statistical or epidemiological authors and $6(28.6 \%)$ IPD-NMAs had a priori protocol. These IPD-NMAs included a median of 19 RCTs, a median of 7110 participants, and evaluated a median of six interventions. The details of the general characteristics are presented in Table 1. 


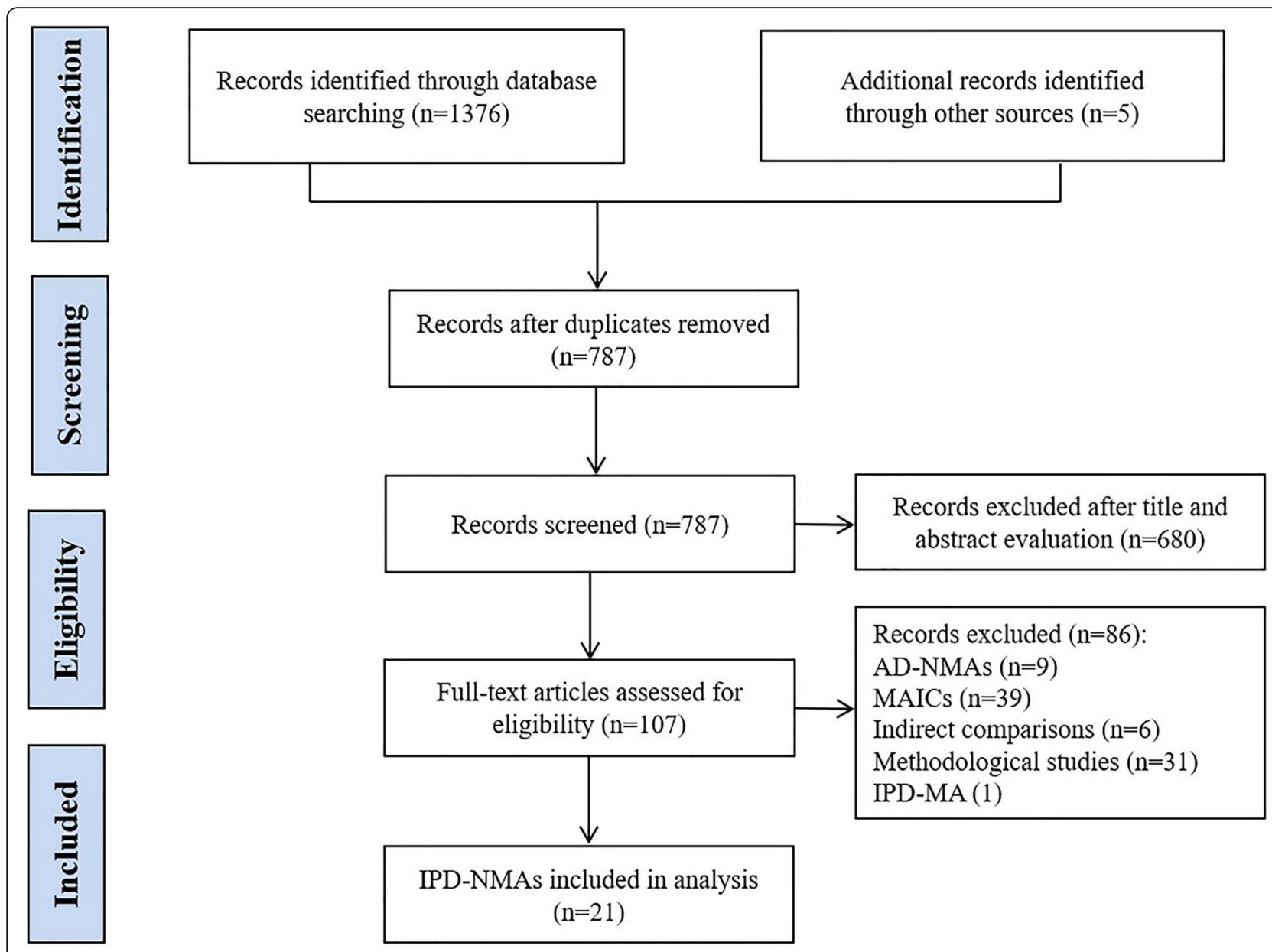

Fig. 1 The flowchart of the screening process. AD-NMAs, aggregate data network meta-analyses; MAICs, matching adjusted indirect comparisons; IPD-MAs, individual patient data meta-analyses; IPD-NMAs, individual patient data network meta-analyses

\section{IPD identification process of included IPD-NMAs}

IPD were obtained by forming collaborative research groups in $12(57.1 \%)$ of the included IPD-NMAs, and the collection of IPD was by contacting authors of relevant studies identified through SRs in 7 (33.3\%) IPDNMAs. Three IPD-NMAs reported the proportion of contacted authors who provided IPD, which ranged from $46.8 \%$ to $80.0 \%$. IPD-NMAs that identified relevant trials through SRs conducted the literature search, and the commonly used databases were PubMed/MEDLINE, Cochrane Library, and ClinicalTrials.gov (Table 2).

\section{Reporting of statistical analyses of included IPD-NMAs}

Table 3 shows the methods of statistical analyses used in the included IPD-NMAs. Fourteen (66.7\%) IPDNMAs used the 1-stage process, and 7 (33.3\%) applied the 2-stage process. Twelve (57.1\%) IPD-NMAs used a Bayesian method, of which 11 synthesized data using the 1-stage process, and nine (42.9\%) used a Frequentist method, of which 6 adopted the 2-stage process. Of the 11 IPD-NMAs included only IPD, 6 applied the Bayesian method, and of the 10 IPDNMAs incorporated both IPD and aggregate data, 4 applied the Frequentist method. None of the IPDNMAs clarified the detailed method used for combining IPD with aggregate data. Of the 12 Bayesian IPDNMAs, all assessed the fit of the model used in data analysis and deviance information criterion (DIC) was the most commonly used method, four did not report the information on the prior distribution, five used the noninformative prior, one used the informative prior, and two adopted the noninformative prior and informative prior. Majority (76.2\%) of the IPD-NMAs simply ignored the missing data, three studies adopted the last observation carried forward (LOCF) to handle the missing data, one used the available case analysis (ACA) method, and one used the Markov chain Monte Carlo (MCMC) multiple imputations method. Heterogeneity was assessed in 15 (71.4\%) IPD-NMAs, and subgroup analysis and sensitivity analysis were the commonly used methods to explore the sources of heterogeneity. However, only nine IPD- 


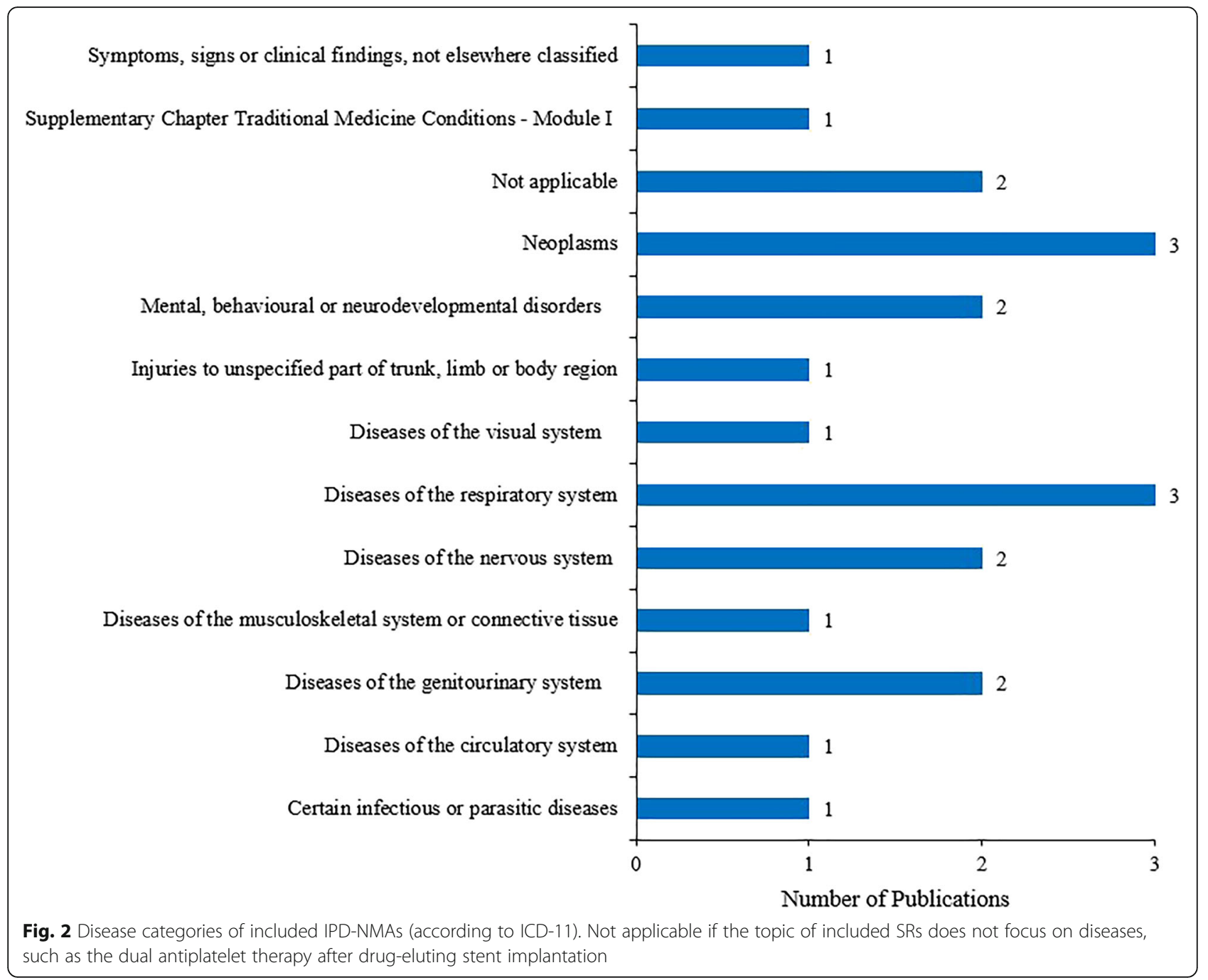

NMAs evaluated the consistency between direct and indirect evidence and none assessed the transitivity.

\section{Reporting quality of included IPD-NMAs}

In terms of the four PRISMA-IPD additional items, the rate of full compliance was $81.0 \%, 52.4 \%, 38.1 \%$, and $14.3 \%$, respectively, for implication, exploration of variation, clarification of the IPD integrity in the "Methods" section, and present it in the "Results" section. Regarding the five PRISMA-NMA supplemental items, one item (S3. Presentation of network structure) was fully reported in $57.1 \%$ of the IPD-NMAs. However, only $14.3 \%$ of the IPD-NMAs described the geometry of network in the "Methods" section and summarized the network geometry in the "Results" section (Fig. 3). Of the remaining 27 items of PRISMA-IPD, the compliance rates of 8 items were higher than $75.0 \%$, and two items (item 3: Rationale, item 26: Conclusions) obtained compliance rates of $100.0 \%$. However, 11 items were not reported in more than $60.0 \%$ IPD-NMAs, and 2 items (item 5: Protocol and registration, item 15: Risk of bias across studies) were only presented in 9.5\% of the IPDNMAs (Additional file 2: Appendix Fig. 1).

IPD-NMAs with a statistician or epidemiologist had a significantly higher rate in reporting exploration for inconsistency $(71.4 \%$ vs. $14.3 \%)$ than other IPD-NMAs. A priori protocol was significantly associated with reporting quality in terms of "search" (88.3\% vs. $26.7 \%)$, "data collection process" (66.7\% vs. $13.3 \%)$, and "results of syntheses" (83.3\% vs. 13.3\%). Except for a significant association between the 1-stage process and "data items," no statistically significant differences were observed between the reporting quality and other factors, including industry funding or not, and Bayesian or Frequentist method (Additional file 1: Appendix Table 1). Improvements were observed in the overall reporting quality (compliance rates of sixteen PRISMA-IPD items and five PRISMA-NMA supplemental items have increased) after 
Table 1 Characteristics of included IPD-NMAs

\begin{tabular}{lll}
\hline tems & Frequency & Proportion (\%) \\
\hline Publication year & 1 & \\
2007 & 1 & 4.8 \\
2010 & 1 & 4.8 \\
2011 & 5 & 4.8 \\
2012 & 1 & 23.8 \\
2013 & 4 & 4.8 \\
2014 & 2 & 19.0 \\
2015 & 3 & 9.5 \\
2017 & 2 & 14.3 \\
2018 & 1 & 9.5 \\
2019 & 1 & 4.8
\end{tabular}

Country of the

correspondence author

$\begin{array}{lll}\text { USA } & 9 & 42.9 \\ \text { UK } & 7 & 33.3 \\ \text { France } & 3 & 14.3 \\ \text { Cameroon } & 1 & 4.8 \\ \text { Netherland } & 1 & 4.8\end{array}$

Journal impact factor

$\begin{array}{lll}0.0 \text { to } 3.0 & 6 & 28.6 \\ 3.1 \text { to } 6.0 & 5 & 23.8 \\ 6.1 \text { to } 15.0 & 2 & 9.5 \\ >15.0 & 6 & 28.6 \\ \text { Non-SCl } & 2 & 9.5\end{array}$

Number of authors

$$
\begin{aligned}
& 1 \text { to } 3 \text { authors } \\
& 4 \text { to } 6 \text { authors } \\
& 7 \text { to } 10 \text { authors } \\
& 11 \text { or more authors }
\end{aligned}
$$

With statistician or epidemiologist

Authors from 2 or more countries

With a priori protocol

Format of data

$$
\begin{aligned}
& \text { IPD only } \\
& \text { IPD + AD }
\end{aligned}
$$

Number of RCTs included: median (IQR)

Number of samples included: median (IQR)

Number of interventions included: $6(4,7)$ median (IQR)

Funding sources

$$
\begin{aligned}
& \text { Industry } \\
& \text { Non-industry }
\end{aligned}
$$

\begin{tabular}{|c|c|c|}
\hline Items & Frequency & $\begin{array}{l}\text { Proportion } \\
\text { (\%) }\end{array}$ \\
\hline \multicolumn{3}{|c|}{ Methods used to identify IPD eligible studies $(n=21)$} \\
\hline Collaborative group ${ }^{a}$ & 12 & 57.1 \\
\hline $\begin{array}{l}\text { Systematic review and contacting } \\
\text { authors }\end{array}$ & 7 & 33.3 \\
\hline Other methods ${ }^{\mathrm{b}}$ & 2 & 9.5 \\
\hline
\end{tabular}

Table 1 Characteristics of included IPD-NMAs (Continued)

\begin{tabular}{lll}
\hline Items & Frequency & Proportion (\%) \\
\hline Industry + non-industry & 3 & 14.3 \\
Unfunded & 1 & 4.8 \\
\hline
\end{tabular}

$S C I$ science citation index, IPD individual participant data, $A D$ aggregate data, IQR interquartile range

Table 2 IPD identification process of included IPD-NMAs

Did the authors obtain IPD from all studies or just a subset? $(n=21)$

$\begin{array}{lll}\text { All studies } & 7 & 33.3 \\ \text { Not reported } & 14 & 66.7\end{array}$

IPD-NMAs that identified IPD through systematic reviews $(n=7)$

Proportion of contacted authors provided IPD

$\begin{array}{lll}46.8 \% & 1 & 14.3 \\ 70.0 \% & 1 & 14.3 \\ 80.0 \% & 1 & 14.3 \\ \text { Not reported } & 4 & 57.1 \\ \text { Whether a literature search was } & 7 & 100.0\end{array}$

conducted? (yes)

Number of databases searched

$\begin{array}{lll}2 \text { to } 5 & 3 & 42.9 \\ 6 \text { to } 9 & 3 & 42.9 \\ 14 & 1 & 14.3\end{array}$

Name of database

PubMed/MEDLINE

85.7

Cochrane Library

85.7

ClinicalTrials.gov

85.7

EMBASE

57.1

Web of Science

28.6

ICRTP

28.6

Reported the year of retrieval of $\quad 6 \quad 85.7$ databases

$\begin{array}{lll}\text { Presented search strategy } & 3 & 42.9 \\ \text { Online supplement } & 1 & 14.3 \\ \text { Manuscript } & 1 & 14.3 \\ \text { Previous published study } & 1 & 14.3\end{array}$

IPD individual participant data, IPD-NMAs individual participant data network meta-analyses, ICRTP World Health Organization International Clinical Trials Registry Platform

a IPD-NMAs project team included authors of IPD studies

${ }^{b}$ Other methods mean obtaining IPD from the Yale Open Data Access Project and a previous meta-analysis 
Table 3 Reporting information of statistical analyses of included IPD-NMAs

\begin{tabular}{ll}
\hline Items & Frequency \\
& $\begin{array}{l}\text { Proportion } \\
(\%)\end{array}$ \\
\hline
\end{tabular}

Fixed- or random-effects?

28.6

Fixed-effects

Random-effects

Fixed- and random-effects

6

Not reported

\section{6}

28.6

28.6

$3 \quad 14.3$

Bayesian or Frequentist method?

Bayesian
Frequentist
1-stage or 2-stage process?
1-stage
2-stage
How was the model fit assessed?

How was the model fit assessed?

\section{Deviance information criterion}

Deviance information criterion + residual deviance

Not reported

Not applicable

Were the prior distributions reported?

Yes

$$
\text { Noninformative prior }{ }^{a}
$$

Informative prior ${ }^{a}$

Noninformative prior + informative prior 2

Not reported

Not applicable

Was the convergence assessed?

Yes

$$
\text { Gelman-Rubin statistic }
$$

Visual plot inspection

Gelman-Rubin statistic + visual plot inspection

Not reported

Not applicable

Statistical techniques used for missing participant data

$$
\begin{aligned}
& \text { LOCF } \\
& \text { ACA }
\end{aligned}
$$

MCMC multiple imputations

Not reported

Was the heterogeneity assessed?

$$
\text { Yes }
$$

\begin{tabular}{|c|c|c|}
\hline Items & Frequency & $\begin{array}{l}\text { Proportion } \\
\text { (\%) }\end{array}$ \\
\hline Node-splitting & 2 & 9.5 \\
\hline Lu and Ades & 1 & 4.8 \\
\hline Lumley & 1 & 4.8 \\
\hline Informal approaches ${ }^{b}$ & 3 & 14.3 \\
\hline Not reported & 12 & 57.1 \\
\hline Was the transitivity assessed? (yes) & 0 & 0 \\
\hline Subgroup analysis conducted? (yes) & 10 & 47.6 \\
\hline Sensitivity analysis conducted? (yes) & 16 & 76.2 \\
\hline Meta-regression analysis conducted? (yes) & 8 & 38.1 \\
\hline With GRADE used & 2 & 9.5 \\
\hline \multicolumn{3}{|l|}{ Software used } \\
\hline WinBUGS/OpenBUGS & 7 & 33.3 \\
\hline $\mathrm{R}$ & 3 & 14.3 \\
\hline WinBUGS + R & 3 & 14.3 \\
\hline WinBUGS + Stata & 1 & 4.8 \\
\hline SAS + Review Manager ${ }^{c}$ & 2 & 9.5 \\
\hline SAS + Stata & 1 & 4.8 \\
\hline $\mathrm{R}+$ Stata & 1 & 4.8 \\
\hline Not reported & 3 & 14.3 \\
\hline
\end{tabular}

Was the consistency assessed?

$$
\text { Yes }
$$

Loop-specific approach
Table 3 Reporting information of statistical analyses of included IPD-NMAs (Continued)

IPD-NMAs individual participant data network meta-analyses, LOCF last observation carried forward, ACA available case analysis, MCMC Markov chain Monte Carlo, GRADE Grading of Recommendations Assessment, Development and Evaluation

aPriors are based on the detailed methods of prior distribution reported in IPD-NMAs

bInformal approaches are the comparison of IPD-NMA with meta-regression IPD-NMA results, comparison of IPD-NMA with aggregate data NMA results, and comparison of NMA results with pairwise meta-analyses results

${ }^{c}$ Review Manager was used for pairwise meta-analyses

the publication of PRISMA-IPD and PRISMA-NMA checklists (Additional file 1: Appendix Table 2).

\section{Methodological quality of included IPD-NMAs}

Of the 21 IPD-NMAs, one was rated as high quality, one was classified as low quality, and the remaining nineteen were rated as critically low quality. Figure 4 shows the compliance rate of each AMSTAR-2 item. $85.7 \%$ of the IPD-NMAs clarified the components of PICO (population, intervention, comparison, and outcome) in the research question and inclusion criteria sections and $71.4 \%$ of the IPD-NMAs used appropriate methods (used an appropriate weighted technique, including the 1-stage or 2-stage, fixed- or random-effects model, and effect measures to combine study results, and investigated the causes of the heterogeneity) for the statistical combination of results. However, none of the IPDNMAs reported the sources of funding for the studies included in the review, only two of the 21 IPD-NMAs pre-specified the review methods and justified the 


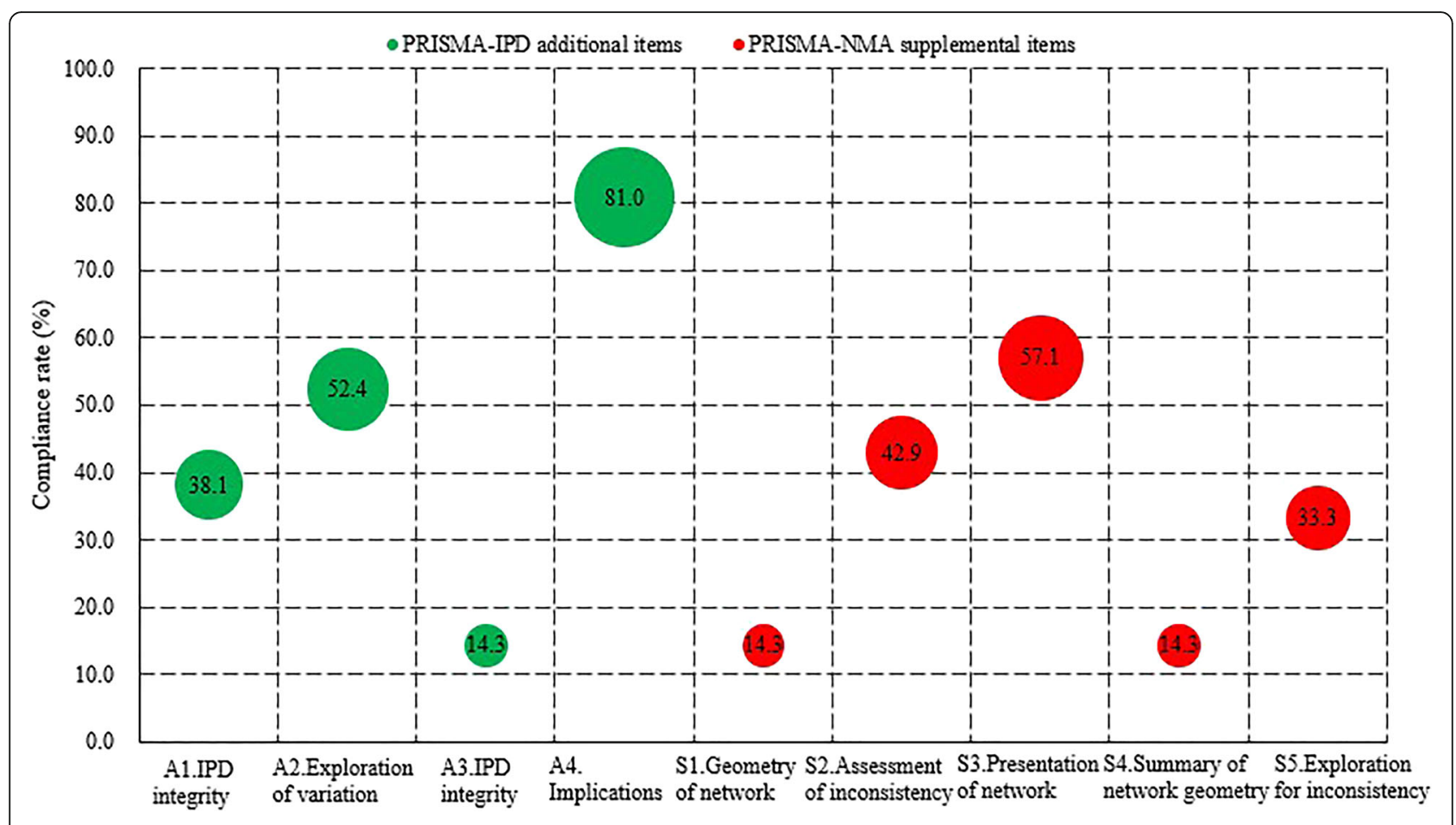

Fig. 3 The full compliance rate of each PRISMA-IPD additional item and PRISMA-NMA supplemental item

13. Consideration of $\mathrm{RoB}$

when interpreting the results 19.0

12.Assess the potential impact of RoB in individual studies

16. Report the conflict of interest 38.1

15.Assessment of presence and likely impact of publication bias 14.3

14.Explain and discuss heterogeneity

47.6

11.Appropriateness of metaanalytical methods

10. Report the sources of 0.0 funding for individual studies
1.Include the components of

PICO

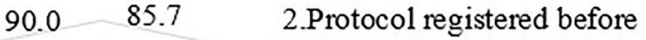

80.0 commencement of the review

$70.0 \quad 9.5$

3. Explain the selection of the

4.8 study designs

4.Adequacy of the literature 52.4 search

5.Perform study selection in 28.6 duplicate

6.Perform data extraction in 33.3 duplicate

19.07 Justification for excluding individual studies

57.1 8.Describe the included studies in adequate detail

9.RoB from individual studies being included in the review

Fig. 4 The full compliance rate of each item based on AMSTAR-2. RoB, risk of bias 
significant deviations from the protocol, and only one explained the selection of the study designs for inclusion in the review. Furthermore, only a few of the included IPD-NMAs provided a list of excluded studies, assessed the potential impact of risk of bias ( $\mathrm{RoB})$ in individual studies on the results of the meta-analysis, and interpreted the results considered the RoB of primary studies (Fig. 4).

Stratified analyses found no statistically significant associations between methodological quality based on the AMSTAR-2 tool and the general characteristics or statistical methods used in IPD-NMAs, including the industry funding or not, pre-specified protocol or not, Bayesian or frequentist method used, and 1-stage or 2-stage process (Additional file 1: Appendix Table 3). There were no significant improvements in the methodological quality of IPD-NMAs after the publication of the AMSTAR-2 tool (Additional file 1: Appendix Table 4).

\section{Result of correlation analysis}

A strong positive correlation was found between the fully reported PRISMA-IPD items and fully reported AMSTAR-2 items (Pearson's $\rho=0.905, P=0.000$ ) as shown in Additional file 2: Appendix Fig. 2.

\section{Discussion}

\section{Findings and interpretations}

This study included 21 IPD-NMAs published between 2007 and 2019, identified from a comprehensive literature search. We investigated the statistical analysis methods used and assessed the reporting quality based on PRISMA-IPD and PRISMA-NMA checklists and methodological quality using the AMSTAR-2 tool. Overall, the reporting of statistical analysis methods was suboptimal, and the reporting quality and methodological quality were low. Compliance rates were insufficient for most of the items of PRISMA-IPD, PRISMA-NMA, and AMSTAR-2.

Of the 21 IPD-NMAs, 10 combined both individual participant data and aggregate data, because IPD were not available from some relevant trials. We also found that the Bayesian IPD-NMAs were more likely to use the 1-stage process, and the Frequentist IPD-NMAs were more likely to use the 2-stage process. Reluctance to share data is a major obstacle to obtaining IPD and performing IPD meta-analysis [36, 37]. A previous study showed that IPD sharing may depend on study characteristics, including funding type, study size, study risk of bias, and treatment effect [27]. Of the 21 included IPDNMAs, only three mentioned the retrieval bias and none assessed the impact of the retrieval bias. One of the advantages of IPD meta-analyses is that it allows the application of appropriate multiple imputation techniques to solve problems related to missing data [3]. However, only $23.8 \%$ of the IPD-NMAs reported the use of statistical techniques to handle missing participant data. Meta-analyses often have intrinsic heterogeneity, which can affect the reliability and validity of results [38]. Heterogeneity was assessed in $71.4 \%$ of the IPD-NMAs, and the sources of heterogeneity were mostly explored by performing the subgroup analysis, meta-regression analysis, or sensitivity analysis. The consistency assumption is imperative for a valid NMA, and the assessment of inconsistency should be fully reported in NMAs [24, 33]. However, no more than $45.0 \%$ of the IPD-NMAs used specific methods to evaluate consistency. The transitivity assumption is another important aspect in NMAs [39], but none of the included IPD-NMAs assessed the transitivity, which should be given more attention in the future work.

For reporting quality based on 31 PRISMA-IPD items and 5 PRISMA-NMA supplemental items, compliance rates were above $70.0 \%$ for nine items, lower than $40.0 \%$ for 14 items, and lower than $15.0 \%$ for 6 items. For IPD meta-analyses, the importance of checking and correcting any inaccuracies or errors in the IPD is self-evident [1]. However, more than $60.0 \%$ of IPD-NMAs did not explore data integrity and about $85.7 \%$ of IPD-NMAs did not report data integrity. Therefore, future IPDNMAs should clarify which aspects of IPD were subject to data checking, how data checking was done, and report any important issues identified in checking IPD. IPD meta-analyses are particularly useful for exploring the participant-level variation of treatment response [1, 40]. Only about half of the included IPD-NMAs described methods for exploring variation in effects by the study- or participant-level characteristics and stated participant-level characteristics that were analyzed as potential effect modifiers. About 86.0\% of IPD-NMAs did not present the network of geometry nor did they summarize the network geometry, which affected the understanding of NMAs [24, 41]. Furthermore, deficiencies were also identified in items related to protocol and registration, search, study selection, data collection process, risk of bias in individual studies, results of syntheses, risk of bias across studies, and funding.

Of the 16 individual AMSTAR-2 items, only four items obtained compliance rates higher than $50.0 \%$, and seven obtained compliance rates lower than $20.0 \%$. Only $4.8 \%$ of the IPD-NMAs explained their selection of the study designs for inclusion in the review, $19.0 \%$ provided a list of excluded studies and justified the exclusions, and none of the IPD-NMAs reported sources of funding for the studies included in the review, which is similar to the findings of other types of SRs and meta-analyses [38, $42,43]$. Therefore, these may be some common methodological shortcomings of any types of SRs. In SRs, assessing the risk of bias of primary studies and 
investigating publication bias are of great importance as these will gauge the validity of meta-analytic results, affect the interpretation of results, and limit our ability to draw conclusions $[43,44]$. Unfortunately, only $28.6 \%$ of IPD-NMAs used a satisfactory technique for assessing the RoB in individual studies included, and only $19 \%$ of IPD-NMAs assessed the potential impact of RoB on the results of the meta-analyses. In addition, only $14.3 \%$ of the included IPD-NMAs investigated the publication bias and discussed its likely impact on the results of the review. Approximately $30.0 \%$ of the IPD-NMAs were industry-sponsored, and no more than $40.0 \%$ of IPDNMAs reported potential conflicts of interest and funding sources, which may lead to potential risks of funding bias [21]. Publishing protocols can reduce the risk of researcher bias and outcome reporting bias [45-47]. Empirical studies have also found that a priori protocol can improve the methodological quality of SR $[34,48]$. Our study showed that IPD-NMAs with a priori protocol tended to have higher methodological quality than IPDNMAs without a priori protocol, although the difference was not statistically significant.

\section{Comparison of our findings with other studies}

A previous study found that key methodological and reporting issues such as consistency assumption, study protocol, and statistical approaches for missing participant data were often insufficiently reported in IPD indirect comparison studies [3]. These results were similar to the findings of our analyses of IPD-NMAs. According to our knowledge, two previous studies $[21,23]$ explored the statistical methods, methodological and reporting characteristics of aggregate data NMAs. These two empirical studies found that, of the included NMAs, only 53.0\% assessed inconsistency, $56.0 \%$ explored heterogeneity, and less than $40.0 \%$ investigated publication bias, which are also similar to the results of our study. This suggested that IPDNMAs and aggregate data NMAs may have the same defects. Compared with Cochrane NMAs [24], IPDNMAs had significantly lower compliance rates in 12 AMSTAR-2 items and 13 PRISMA-NMA items, revealing that both the methodological and reporting quality of IPD-NMAs were lower than Cochrane NMAs. Therefore, there was room for further improvement in both Cochrane NMAs and IPD-NMAs, such as the geometry of the network, the risk of bias in individual studies, and the assessment of inconsistency.

\section{Strengths and limitations}

This is the first comprehensive evaluation of published IPD-NMAs, in terms of statistical methods used, reporting quality based on PRISMA-IPD and PRISMA-NMA checklists, and methodological quality based on the AMSTAR-2 tool. Furthermore, we also conducted stratified analyses to explore potential factors that may affect the reporting and methodological quality and further performed the correlation analysis to evaluate whether the reporting quality was relevant to the methodological quality. However, our study also has some limitations. First, the number of IPD-NMAs included in our study was small, although we have identified all available IPD-NMAs by conducting a comprehensive literature search. Second, our data depended on the information reported in the included IPD-NMAs, so we could not rule out the possibility that some important methods were appropriately used in the study but not reported [3]. Third, we mainly focused on the impact of selected factors on the methodological and reporting quality of IPD-NMAs. Finally, we included a very small number of IPDNMAs and conducted a large number of statistical tests. Any significant results of stratified analyses should be interpreted with caution.

\section{Implications for further research and practice}

Our study indicated that the reporting and methodological quality of IPD-NMAs needs to be further improved. The identified drawbacks need to be addressed, and future IPD-NMAs should be conducted according to reporting and methodological guidelines [1, 33, 35]. In this study, we assessed the reporting quality of IPDNMAs using PRISMA-IPD and PRISMA-NMA statements. However, the PRISMA-IPD statement aims to improve the reporting quality of SRs and meta-analyses of IPD, while the PRISMA-NMA aims to improve the completeness and transparency of reporting of NMAs. Currently, there are no reporting standards aimed to enhance the reporting of IPD-NMAs. Therefore, it is necessary to develop a reporting quality tool for IPDNMAs, incorporating relevant items from both PRISMA-IPD and PRISMA-NMA. For example, the report should be identified as a network meta-analysis of individual participant data in the title; describe methods used to explore the network geometry, transitivity assumption, and consistency assumption; and describe statistical techniques used for missing participant data and methods used to explore variation in effects.

\section{Conclusions}

The key information on statistical methods was often missing, and compliance rates of reporting and methodological items were suboptimal in published IPDNMAs. Methodological and reporting shortcomings include handling of missing participant data, assessment of publication bias, clarification of the IPD integrity, description of network geometry, and 
assessment of the consistency. Authors of future IPDNMAs should address the identified flaws and strictly adhere to methodological and reporting guidelines. We recommend the development of a reporting quality tool that is specifically applicable to IPD-NMAs.

\section{Supplementary information}

Supplementary information accompanies this paper at https://doi.org/10. 1186/s12916-020-01591-0.

Additional file 1: Appendix Word 1. Search strategy of PubMed. Appendix Word 2. Result of the normality test. Appendix Word 3. The list of included IPD-NMAs. Appendix Table 1. Stratified analyses of reporting quality assessment in PRISMA-IPD items and PRISMA-NMA supplemental items. Appendix Table 2. Reporting quality between IPDNMAs before and after the publication of PRISMA-IPD and PRISMA-NMA checklists. Appendix Table 3. Stratified analyses of methodological quality assessment in AMSTAR-2 items. Appendix Table 4. Methodological quality between IPD-NMAs before and after the publication of the AMSTAR-2 checklist.

Additional file 2: Appendix Figure 1. The full compliance rate of each PRISMA-IPD item and PRISMA-NMA supplemental item. Appendix Figure 2. Correlation between fully reported PRISMA-IPD items and fully reported AMSTAR-2 items.

\section{Abbreviations}

AMSTAR-2: Assessment of Multiple Systematic Reviews-2; GRADE: Grading of Recommendations Assessment, Development and Evaluation; IPDNMAs: Individual participant data network meta-analyses; PRISMAIPD: Preferred Reporting Items for Systematic Review and Meta-Analyses of individual participant data; PRISMA-NMA: Preferred Reporting Items for Systematic Reviews and Meta-analyses extension; RCT: Randomized controlled trial; SR: Systematic review

\section{Acknowledgments}

None.

\section{Authors' contributions}

YG, FS, and JT planned and designed the study. YG and JT developed search strategies. YG, SS, ML, and XL screened potential studies and extracted data from the included studies. YG, SS, ML, and KY managed the data and performed the statistical analysis. FS and JT conducted the arbitration under disagreement and ensured that there were no errors. JZ, FS, and JT provided methodological support and helped to interpret findings. YG and JT wrote the first draft. YG, FS, and JT revised the draft. The authors approved the final version of the manuscript.

\section{Funding}

None.

\section{Availability of data and materials}

All data generated or analyzed during this study are included in this published article.

\section{Ethics approval and consent to participate}

Not applicable.

\section{Consent for publication}

Not applicable.

\section{Competing interests}

The authors declare that they have no competing interests.

\section{Author details}

${ }^{1}$ Evidence-Based Medicine Center, School of Basic Medical Sciences, Lanzhou University, No.199, Donggang West Road, Lanzhou City 730000, Gansu Province, China. ${ }^{2}$ The Second Clinical Medical College of Lanzhou University, Lanzhou, China. ${ }^{3}$ Evidence-Based Nursing Center, School of Nursing, Lanzhou
University, Lanzhou, China. ${ }^{4}$ Evidence-Based Medicine Center, Tianjin University of Traditional Chinese Medicine, Tianjin, China. ${ }^{5}$ Public Health and Health Services Research, Norwich Medical School, University of East Anglia, Norwich NR4 7TJ, UK. ${ }^{6}$ Key Laboratory of Evidence-Based Medicine and Knowledge Translation of Gansu Province, Lanzhou, China.

Received: 16 January 2020 Accepted: 14 April 2020

Published online: 01 June 2020

\section{References}

1. Stewart LA, Clarke M, Rovers M, Riley RD, Simmonds M, Stewart G, Tierney JF. Preferred reporting items for systematic review and meta-analyses of individual participant data: the PRISMA-IPD statement. JAMA. 2015;313(16): 1657-65.

2. Simmonds $M$, Stewart $G$, Stewart L. A decade of individual participant data meta-analyses: a review of current practice. Contemp Clin Trials. 2015;45(Pt A):76-83

3. Veroniki AA, Straus SE, Soobiah C, Elliott MJ, Tricco AC. A scoping review of indirect comparison methods and applications using individual patient data. BMC Med Res Methodol. 2016:16:47.

4. Schuit $\mathrm{E}, \mathrm{Li} A \mathrm{AH}$, loannidis JPA. How often can meta-analyses of individuallevel data individualize treatment? A meta-epidemiologic study. Int J Epidemiol. 2019;48(2):596-608.

5. Stewart LA, Tierney JF. To IPD or not to IPD? Advantages and disadvantages of systematic reviews using individual patient data. Eval Health Prof. 2002; 25(1):76-97.

6. Tsujimoto Y, Fujii T, Onishi A, Omae K, Luo Y, Imai H, Takahashi S, Itaya T, Pinson C, Nevitt SJ, et al. No consistent evidence of data availability bias existed in recent individual participant data meta-analyses: a metaepidemiological study. J Clin Epidemiol. 2020;118:107-14.e5.

7. Debray TPA, Schuit E, Efthimiou O, Reitsma JB, loannidis JPA, Salanti G, Moons KGM. An overview of methods for network meta-analysis using individual participant data: when do benefits arise? Stat Methods Med Res. 2018;27(5):1351-64.

8. Tierney JF, Vale C, Riley R, Smith CT, Stewart L, Clarke M, Rovers M. Individual participant data (IPD) meta-analyses of randomised controlled trials: guidance on their use. PLoS Med. 2015;12(7):e1001855.

9. Li T, Puhan MA, Vedula SS, Singh S, Dickersin K. Network meta-analysishighly attractive but more methodological research is needed. BMC Med. 2011;9:79.

10. Mills EJ, loannidis JP, Thorlund K, Schunemann HJ, Puhan MA, Guyatt GH. How to use an article reporting a multiple treatment comparison metaanalysis. JAMA. 2012;308(12):1246-53.

11. Caldwell DM, Ades AE, Higgins JP. Simultaneous comparison of multiple treatments: combining direct and indirect evidence. BMJ (Clinical research ed). 2005;331(7521):897-900.

12. Li L, Tian J, Tian H, Moher D, Liang F, Jiang T, Yao L, Yang K. Network metaanalyses could be improved by searching more sources and by involving a librarian. J Clin Epidemiol. 2014;67(9):1001-7.

13. Thom HH, Capkun G, Cerulli A, Nixon RM, Howard LS. Network metaanalysis combining individual patient and aggregate data from a mixture of study designs with an application to pulmonary arterial hypertension. BMC Med Res Methodol. 2015;15:34.

14. Donegan S, Welton NJ, Tudur Smith C, D'Alessandro U, Dias S. Network meta-analysis including treatment by covariate interactions: consistency can vary across covariate values. Res Synth Methods. 2017:8(4):485-95.

15. Donegan S, Williamson P, D'Alessandro U, Garner P, Smith CT. Combining individual patient data and aggregate data in mixed treatment comparison meta-analysis: individual patient data may be beneficial if only for a subset of trials. Stat Med. 2013:32(6):914-30.

16. Saramago P, Sutton AJ, Cooper NJ, Manca A. Mixed treatment comparisons using aggregate and individual participant level data. Stat Med. 2012;31(28): 3516-36.

17. Berlin JA, Santanna J, Schmid CH, Szczech LA, Feldman HI. Individual patient- versus group-level data meta-regressions for the investigation of treatment effect modifiers: ecological bias rears its ugly head. Stat Med. 2002:21(3):371-87.

18. Riley RD, Lambert PC, Abo-Zaid G. Meta-analysis of individual participant data: rationale, conduct, and reporting. BMJ (Clinical research ed). 2010;340: c221 
19. Veroniki AA, Straus SE, Ashoor HM, Hamid JS, Hemmelgarn BR, HolroydLeduc J, Majumdar SR, McAuley G, Tricco AC. Comparative safety and effectiveness of cognitive enhancers for Alzheimer's dementia: protocol for a systematic review and individual patient data network meta-analysis. BMJ Open. 2016;6(1):e010251

20. Simmonds MC, Higgins JPT, Stewart LA, Tierney JF, Clarke MJ, Thompson SG. Meta-analysis of individual patient data from randomized trials: a review of methods used in practice. Clin Trials. 2005;2(3):209-17.

21. Zarin W, Veroniki AA, Nincic V, Vafaei A, Reynen E, Motiwala SS, Antony J, Sullivan SM, Rios P, Daly C, et al. Characteristics and knowledge synthesis approach for 456 network meta-analyses: a scoping review. BMC Med. 2017; 15(1):3.

22. Tonin FS, Borba HH, Leonart LP, Mendes AM, Steimbach LM, Pontarolo R, Fernandez-Llimos F. Methodological quality assessment of network metaanalysis of drug interventions: implications from a systematic review. Int J Epidemiol. 2019;48(2):620-32

23. Petropoulou M, Nikolakopoulou A, Veroniki AA, Rios P, Vafaei A, Zarin W, Giannatsi M, Sullivan S, Tricco AC, Chaimani A, et al. Bibliographic study showed improving statistical methodology of network meta-analyses published between 1999 and 2015. J Clin Epidemiol. 2017;82:20-8.

24. Gao Y, Ge L, Ma X, Shen X, Liu M, Tian J. Improvement needed in the network geometry and inconsistency of Cochrane network meta-analyses: a cross-sectional survey. J Clin Epidemiol. 2019;113:214-27.

25. Chaimani A, Vasiliadis HS, Pandis N, Schmid CH, Welton NJ, Salanti G. Effects of study precision and risk of bias in networks of interventions: a network meta-epidemiological study. Int J Epidemiol. 2013;42(4):1120-31.

26. Jansen JP. Network meta-analysis of individual and aggregate level data. Res Synth Methods. 2012;3(2):177-90.

27. Veroniki AA, Ashoor HM, Le SPC, Rios P, Stewart LA, Clarke M, Mavridis D, Straus SE, Tricco AC. Retrieval of individual patient data depended on study characteristics: a randomized controlled trial. J Clin Epidemiol. 2019:113:176-88.

28. Freeman SC, Fisher D, Tierney JF, Carpenter JR. A framework for identifying treatment-covariate interactions in individual participant data network meta-analysis. Res Synth Methods. 2018;9(3):393-407.

29. Riley RD, Ensor J, Jackson D, Burke DL. Deriving percentage study weights in multi-parameter meta-analysis models: with application to meta-regression, network meta-analysis and one-stage individual participant data models. Stat Methods Med Res. 2018;27(10):2885-905.

30. Signorovitch JE, Wu EQ, Yu AP, Gerrits CM, Kantor E, Bao Y, Gupta SR, Mulani PM. Comparative effectiveness without head-to-head trials: a method for matching-adjusted indirect comparisons applied to psoriasis treatment with adalimumab or etanercept. PharmacoEconomics. 2010; 28(10):935-45.

31. Bucher HC, Guyatt GH, Griffith LE, Walter SD. The results of direct and indirect treatment comparisons in meta-analysis of randomized controlled trials. J Clin Epidemiol. 1997;50(6):683-91.

32. Caro JJ, Ishak KJ. No head-to-head trial? Simulate the missing arms. PharmacoEconomics. 2010;28(10):957-67.

33. Hutton B, Salanti G, Caldwell DM, Chaimani A, Schmid CH, Cameron C, loannidis JP, Straus S, Thorlund K, Jansen JP, et al. The PRISMA extension statement for reporting of systematic reviews incorporating network metaanalyses of health care interventions: checklist and explanations. Ann Intern Med. 2015;162(11):777-84.

34. Ge L, Tian JH, Li YN, Pan JX, Li G, Wei D, Xing X, Pan B, Chen YL, Song FJ, et al. Association between prospective registration and overall reporting and methodological quality of systematic reviews: a meta-epidemiological study. J Clin Epidemiol. 2018;93:45-55.

35. Shea BJ, Reeves BC, Wells G, Thuku M, Hamel C, Moran J, Moher D, Tugwell P, Welch V, Kristjansson E, et al. AMSTAR 2: a critical appraisal tool for systematic reviews that include randomised or non-randomised studies of healthcare interventions, or both. BMJ (Clinical research ed). 2017;358:j4008.

36. Villain B, Dechartres A, Boyer P, Ravaud P. Feasibility of individual patient data meta-analyses in orthopaedic surgery. BMC Med. 2015;13:131.

37. Jaspers GJ, Degraeuwe PL. A failed attempt to conduct an individual patient data meta-analysis. Syst Rev. 2014;3:97.

38. Jiang Q, Liu Q, Chen F, Zeng X, Song F, Lu Z, Cao S. Reporting quality and statistical analysis of published dose-response meta-analyses was suboptimal: a cross-sectional literature survey. J Clin Epidemiol. 2019;115: $133-40$
39. Song F, Loke YK, Walsh T, Glenny AM, Eastwood AJ, Altman DG. Methodological problems in the use of indirect comparisons for evaluating healthcare interventions: survey of published systematic reviews. BMJ (Clinical research ed). 2009:338:b1147.

40. Hingorani AD, Windt DA, Riley RD, Abrams K, Moons KG, Steyerberg EW, Schroter S, Sauerbrei W, Altman DG, Hemingway H. Prognosis research strategy (PROGRESS) 4: stratified medicine research. BMJ (Clinical research ed). 2013:346:e5793.

41. Bafeta A, Trinquart $L$, Seror $R$, Ravaud P. Reporting of results from network meta-analyses: methodological systematic review. BMJ (Clinical research ed). 2014:348:91741.

42. Habtewold TD, Alemu SM, Mohammed SH, Endalamaw A, Mohammed MA, Teferra AA, Tura AK, Asefa NG, Tegegne BS. Biomedical and public health reviews and meta-analyses in Ethiopia had poor methodological quality: overview of evidence from 1970 to 2018. J Clin Epidemiol. 2019:109:90-8.

43. Yan P, Yao L, Li H, Zhang M, Xun Y, Li M, Cai H, Lu C, Hu L, Guo T, et al. The methodological quality of robotic surgical meta-analyses needed to be improved: a cross-sectional study. J Clin Epidemiol. 2019;109:20-9.

44. Riado Minguez D, Kowalski M, Vallve Odena M, Longin Pontzen D, Jelicic Kadic A, Jeric M, Dosenovic S, Jakus D, Vrdoljak M, Poklepovic Pericic T, et al. Methodological and reporting quality of systematic reviews published in the highest ranking journals in the field of pain. Anesth Analg. 2017;125(4): 1348-54.

45. Silagy CA, Middleton P, Hopewell S. Publishing protocols of systematic reviews: comparing what was done to what was planned. JAMA. 2002; 287(21):2831-4.

46. Koensgen N, Rombey T, Allers K, Mathes T, Hoffmann F, Pieper D. Comparison of non-Cochrane systematic reviews and their published protocols: differences occurred frequently but were seldom explained. J Clin Epidemiol. 2019;110:34-41.

47. Page MJ, McKenzie JE, Forbes A. Many scenarios exist for selective inclusion and reporting of results in randomized trials and systematic reviews. J Clin Epidemiol. 2013;66(5):524-37.

48. Allers K, Hoffmann F, Mathes T, Pieper D. Systematic reviews with published protocols compared to those without: more effort, older search. J Clin Epidemiol. 2018;95:102-10.

\section{Publisher's Note}

Springer Nature remains neutral with regard to jurisdictional claims in published maps and institutional affiliations.
Ready to submit your research? Choose BMC and benefit from:

- fast, convenient online submission

- thorough peer review by experienced researchers in your field

- rapid publication on acceptance

- support for research data, including large and complex data types

- gold Open Access which fosters wider collaboration and increased citations

- maximum visibility for your research: over $100 \mathrm{M}$ website views per year

At $\mathrm{BMC}$, research is always in progress.

Learn more biomedcentral.com/submissions 